\title{
Concerning Dediu M, Zielinski A: A Proposal to Redefine Pathologic Complete Remission as Endpoint following Neoadjuvant Chemotherapy in Early Breast Cancer. Breast Care 2019; Doi 10.1159/000500620
}

\author{
Michael Untch $^{\text {a }}$ Sibylle Loibl $^{b}$ Peter A. Fasching ${ }^{c}$ \\ ${ }^{a}$ Clinic for Gynecology, Gynecologic Oncology and Obstetrics, Helios Hospital Berlin-Buch, Berlin, Germany; \\ ${ }^{b}$ Medicine and Research, GBG Forschungs GmbH, Neu-Isenburg, Germany; \\ 'Frauenklinik, Universitätsklinikum Erlangen, Erlangen, Germany
}

We highly acknowledge the effort of our colleagues Dediu and Zielinski to redefine pathologic complete remission as endpoint following neoadjuvant chemotherapy (NACT) in patients with early breast cancer.

One critical question has to be answered first. Is a pathological complete response (pCR) related to improved survival?

We have to congratulate those pioneers in the breast cancer field who had the courage in the 1980s of the last century to perform prospective randomized trials showing that those patients who had a pCR after NACT had an excellent outcome [1]. After the first generation of NACT trials, new studies including monoclonal antibodies as targeted therapy against HER2 showed a dramatic increase in the pCR rate from $<20 \%$ to about $40-45 \%$, and this again resulted in a significant increase in disease-free (DFS) and overall survival (OS). Therefore, the conclusion was that $\mathrm{pCR}$ is an excellent surrogate marker for long-term outcome in this patient group [2,3]. The largest worldwide meta-analysis of patients treated in prospective randomized trials with NACT demonstrated an excellent correlation of pCR with DFS and OS, especially in patients who had triple-negative or HER2-positive tumors [4]. The St. Gallen Consensus Meeting panelists opted in the year 2017 and 2019 again with a large majority for NACT with anti-HER2 treatment as a preferred option in patients with HER2-positive early breast cancer and for NACT in patients with triple-negative tumors [5]. NACT has the potential to accelerate the progress in the medical treatment of breast cancer patients by using $\mathrm{pCR}$ as a surrogate marker for DFS and OS [6,7].

We have also learned some critical lessons from neoadjuvant trials. One of them is that pCR is not a good surrogate marker for outcome in patients who have an invasive lobular cancer and receive NACT [8].

Results from NACT trials will also foster further development to optimize breast cancer therapy. We have shown that patients who have pCR have an excellent prognosis. Those patients who had residual invasive tumor cells in the breast and/or in the ipsilateral axillary nodes and have Ki- $67<15 \%$ have a DFS similar to patients who had a pCR. On the other hand, those patients who had Ki-67 $>35 \%$ in their residual tumor after NACT have a significantly worse DFS [9].

The knowledge about pCR or non-pCR after NACT has triggered a new generation of postneoadjuvant trials; the first one, a study from Japan, has shown a survival benefit in those patients who had NACT with anthracyclines and taxanes. The survival benefit was seen especially in patients with triple-negative breast cancer (TNBC) who had residual disease in the breast and/or in the ipsilateral axillary lymph nodes and received additional capecitabine chemotherapy after NACT [10]. This has also led to a strong recommendation by $>90 \%$ of the 
International St. Gallen Committee members in 2017 and 2019 for postneoadjuvant capecitabine in patients with $\mathrm{TNBC}$ and residual tumor $>1 \mathrm{~cm}$ or positive nodes after NACT [5].

In the newest generation of postneoadjuvant trials like the KATHERINE study, patients with HER2-positive tumors and residual tumor in the breast and/or lymph nodes after optimal NACT including anti-HER2 therapy were randomized to standard anti-HER2 postoperative treatment versus treatment against HER2 with the antibody conjugate T-DM1. The study has been recently reported at the San Antonio Breast Cancer Conference in December 2018 and published in the New England Journal of Medicine [11]. The application of T-DM1 in patients with residual tumor demonstrated a statistically significant and clinically meaningful improvement in invasive DFS (IDFS) compared with trastuzumab (unstratified HR $=0.50 ; 95 \%$ CI 0.39-0.64; $p<0.0001)$. The 3-year IDFS increased from 77.0 to $88.3 \%$ (difference $=11.3 \%$ ). The IDFS benefit was consistent across all subgroups including HR status, extent of residual invasive disease, and single or dual HER2-targeted neoadjuvant therapy (NAT). These data will be the basis of the new standard of care in this patient population and should increase the use of NAT in patients with HER2-positive early-stage breast cancer $[11,12]$.

The PENELOPE-B trial randomized patients with HER2-negative hormone receptor-positive tumors who had residual tumor after NACT and a CPS-EG score $\geq 3$ or 2 and nodal involvement to standard adjuvant endocrine treatment versus standard adjuvant endocrine treatment with the addition of the CDK 4/6 inhibitor palbociclib [13].

The third new generation study is OLYMPIA, in which patients with germline BRCA mutations and residual disease after NACT are randomized to the BRCA-targeted (e.g., homologous repair deficiency) therapy with olaparib.

\section{Summary}

- $\mathrm{pCR}$ is an excellent surrogate marker for survival, especially in patients with TNBC or HER2 overexpression.

- $\mathrm{pCR}$ is less reliable as a surrogate marker for outcome in patients with lobular cancer.

- The area between pCR and non-pCR should be explored in studies.

- In patients with residual invasive tumor in the breast and/or lymph nodes (non-pCR patients), the biology of the residual tumor should be further explored.

- Non-pCR (residual disease in the breast and especially in the ipsilateral lymph nodes) will guide us to tailor further treatment (radiotherapy and medical treatment according to biology).
A further major advantage of NACT is less radical surgery with more breast- and axillary lymph node-conserving therapy.

Most of the patients who are candidates for NACT have a chance of about $70 \%$ to have breast-conserving surgery. Analysis of 5,477 breast cancer patients after NACT has shown that not the type of surgery is mainly influencing locoregional recurrence (LRR)-free survival, but the biology of the tumor; the highest LRR incidence occurred in patients with TNBC or a HER2-overexpressing tumor, who did not receive anti-HER2 treatment and had residual disease in the breast and especially in the lymph nodes after NACT [14]. The pooled analysis results from the collaborative trials in neoadjuvant breast cancer (CTNeoBC) show similar results. Out of 5,250 patients, 391 had LRR after NAT and surgery. In 63/1,187 patients, these LRR occurred in patients who had pCR compared to $107 / 1,827$ patients who had residual tumor in the breast and 221/2,238 patients who had residual tumor in the ipsilateral axillary lymph nodes. The highest LRR incidence was seen in patients younger than 50 years with a grade 3 tumor and residual disease in the lymph nodes ( 20.6 vs. $2.8 \%$ LRR in patients with a grade $1-2$ tumor and no invasive residual disease after NACT). The incidence of LRR was highest (35\%) in patients with a hormone receptor-negative HER2-positive tumor $<50$ years with residual tumor in the breast after NACT. This high LRR was mainly triggered by studies without neoadjuvant anti-HER2 treatment. Even in patients who had mastectomy, LRR was highest in patients with HER2positive tumors if these patients had residual tumor after NACT in the ipsilateral lymph nodes $(24.4 \%$ in ypN+ compared to $4.1 \%$ in patients with pCR) [15]. In more recent studies, LRR has decreased and is also affected by tumor biology and response to NAT [16].

According to the national German guidelines, the pretherapeutic assessment includes palpation, mammography, ultrasound of the breast and the axilla, minimally invasive biopsy, and, if indicated, magnetic resonance imaging [17].

The recommended methods for monitoring the response during and after neoadjuvant systemic chemotherapy are: breast ultrasound, palpation, mammography, MRI, and sometimes PET/CT. It is mandatory to clip the tumor region before NACT to guide surgery after NAT. In order to have targeted surgery after NAT, the recommendation is to prove microscopically clear resection margins in pathology. Tumor resection in the new (shrinked) borders is safe [17]. The published studies performed by the AGO-B and GBG study groups have paid an extensive amount of attention to this fact. A multidisciplinary team has to review imaging and the clinical situation before and during NAT and before surgery. The pathohistological result after surgery has to be correlated 
in the team especially with breast and axillary imaging. Today, we also have localization techniques which increase the rate of breast-conserving surgery, like intraoperative ultrasound, and wire- and radio-guided lesion localization. There are clear recommendations for the standardized pathologic characterization of residual disease after NACT [18].

Two large prospective randomized trials have analyzed the role of sentinel lymph node biopsy in patients who underwent NACT. One was the SENTINA trial from Germany [19] and the other the ACOSOG Z1071 trial [20]. Both studies have set the basis for the implementation of sentinel node biopsy in the context of NACT and aim to reduce the radicality of axillary surgery after NACT.

These studies have resulted in a change of the German national guideline for axillary intervention before and after NACT: for patients who have unsuspicious ipsilaterally lymph nodes by palpation and sonography, we recommend sentinel lymph node biopsy after and not before NACT. In patients who convert from clinically and sonographically suspicious lymph nodes (proven by biopsy to be positive) to clinically/sonographically lymph node negative, we recommend the sentinel lymph node biopsy after NACT. If those sentinel lymph nodes prove to be positive, axillary lymph node dissection is still advised. In those patients who have a clip placement before NAT in the histologically involved lymph node and who convert to clinically and sonographically negative axillary lymph nodes, there are increasing data to refrain from full axillary dissection if 2 or 3 lymph nodes are not histologically positive for tumor cells [21]. Further studies address the question of targeted axillary dissection with clip or radiocolloid localization after NACT [21, 22].

Today, we even go one step further and ask the question whether it is feasible to skip breast cancer surgery completely if we can reliably recognize those patients who have a pCR. One of the first attempts was very encouraging, showing that in patients who had multiple vacuumassisted core biopsies and fine-needle aspiration after NACT with good clinical and imaging response, the predictive accuracy for pCR was 98\% [23].

One of the next steps in the de-escalation of treatment in patients with pCR after NACT is decreasing the extent of locoregional radiotherapy. This is the approach in the ongoing NSABP B51/RTOG 1,304 study.

In a recent meta-analysis of 4,125 patients, the following factors where associated with high-risk LRR: $\mathrm{CN} 1$ involvement at presentation, residual tumor in the ipsilateral axillary lymph nodes, and hormone receptor-negative tumors at presentation [24]. Especially those patients who convert from lymph node positive to lymph node negative by NAT have a significant benefit in terms of LRR $[24,25]$. Although modern locoregional radiothera- py de-escalation studies deal with this topic, we should still use radiotherapy after NACT as indicated in the national and international guidelines [26].

The subtitle 2 on page 5 "Is $p$ CR following NAT prognostic or predictive?" is misleading. Up to now, most of the studies including the meta-analysis of Cortazar et al. [4] have shown that $\mathrm{pCR}$ is an excellent surrogate marker for DFS and OS especially in patients with TNBC or HER2overexpressing tumors. Since pCR is the result of an intervention (namely NACT or NACT with additional targeted therapy), the question of being prognostic or predictive cannot be addressed.

The question of whether pCR is predictive can be addressed in non-pCR patients with an additional intervention like post-NAT and has to demonstrate a positive outcome of this second intervention. The Create-X [10] and the KATHERINE study [11] are the blueprint for this hypothesis and have unambiguously demonstrated that pCR is not just prognostic but even more predictive for the future outcome of patients with non-pCR by a second intervention which is specific post-NAT (capecitabine for patients with HER2-negative tumors and T-DM1 for patients with HER2-positive tumors). Further studies which are addressing this question are either finished or ongoing and have been addresses previously (PENELOPE-B, and OLYMPIA study).

The sentence "Despite the mentioned important considerations regarding the efficacy of treatment modalities for early breast cancer, we believe that using pCR as a surrogate endpoint for OS in NAT trials refers to the prognostic rather than predictive relevance of $\mathrm{pCR}$, with the latter being only marginal" would be true only in the case that postneoadjuvant trials fail. Therefore, we ask the authors to focus on these postneoadjuvant trials as a critical point to prove the hypothesis that $\mathrm{pCR}$ after NAT is not only prognostic but also predictive.

\section{Prognostic Significance of pCR following NAT}

It is true that patients included in the mentioned metaanalysis of Cortazar et al. [4] are coming from a mixed population comprising primarily node-negative but also node-positive patients. A split made by the authors of this article between node-negative and node-positive patients with 1-3 and $>4$ involved lymph nodes is also a little bit misleading, because from studies on the tumor biology by multigene signatures we know that there are patients with uninvolved and 1- to 3-involved lymph nodes who are at a very high risk of metastasis and need additional systemic therapy like chemotherapy and/or targeted therapy. The fact that a part of the patients in neoadjuvant trials had node-negative disease and a part had node-positive disease is very well acknowledged, and we also know that 
many patients become node negative after NACT (see previous comments on axillary intervention before and after NACT).

\section{Results of Randomized Trials in HER2-Positive and TNBC Subsets}

It is correct that despite a significant improvement in pCR in the neoadjuvant neoALTTO trial following combined therapy with trastuzumab/lapatinib and chemotherapy did not translate into a better survival compared to standard trastuzumab treatment combined with chemotherapy in the adjuvant ALTTO study. The study populations of these two studies are completely different. The neoALTTO study included patients with larger tumors and especially higher tumor burden in the axillary nodes, whereas in the ALTTO study $30 \%$ of the patients had uninvolved lymph nodes and smaller tumors. These patients have already an excellent outcome after standard therapy and do not need a double blockade with a receptor tyrosine kinase inhibitor and trastuzumab. On the other hand, the GeparQuinto study [27] has shown that especially in patients with hormone receptor-positive tumors the addition of a receptor tyrosine kinase inhibitor to trastuzumab translates into a significantly better outcome, which has also been shown by the ExteNET study with neratinib [28]. Therefore, one should not mix both studies in the argumentation. The neoALTTO study stands for itself, and the ALTTO study has indeed a similar design but a completely different patient population. The duration of the treatment with an anti-HER2 antibody and a receptor tyrosine kinase inhibitor is different in these studies.

The same argument is true for the neoadjuvant NeoSphere and the adjuvant APHINITY studies mentioned by the authors: NeoSphere tested the addition of pertuzumab to the trastuzumab NACT backbone and showed a significant improvement in pCR. The study has a different population than the APHINITY study in which $30 \%$ of patients were node negative. In these patients, there was no significant benefit of the combination of 2 targeted antibodies trastuzumab and pertuzumab. On the other hand, in the APHINITY study, this double antibody combination showed a significant benefit especially in node-positive and hormone receptor-negative patients, which led to the approval of this combination by the FDA and by the European Medical Agency.

The CALGB 40603 study cited could indeed not show a significant increase in DFS in patients with TNBC although pCR was increased by the addition of carboplatin to NACT. Unfortunately, this trial was not powered for DFS, but the trend was clear with 76 versus $71 \%$ DFS with the addition of carboplatin. The German GeparSixto trial clearly demonstrated a significant increase in DFS by the addition of carboplatin to a neoadjuvant anthracyclinetaxane-containing chemotherapy [29].

The conclusions of the authors: "to use this endpoint in NAT trials may select for inappropriate, possibly more toxic regimens while discarding potentially efficient therapies" is also misleading. To the contrary, we should learn the lesson of escalation and de-escalation, especially from neoadjuvant studies.

The authors of this review have tried several times to de-escalate therapy in patients with pCR, e.g., discontinuation of postneoadjuvant anti-HER2-therapy in patients achieving pCR after NAT including anti-HER2-therapy. This point has never been addressed in a study because pharma industry-driven studies do not like this type of de-escalation.

On the other hand, postneoadjuvant trials try to prove that patients who did not achieve pCR need escalation of treatment. A first example is the Create-X study from Japan which showed a significant DFS and OS benefit in patients treated with additional post-NACT with 6 cycles of capecitabine if they did not achieve pCR [10] and the KATHERINE study recently presented in San Antonio and published in the NEJM [11]. These studies underline the importance of pCR for escalation and de-escalation.

Moreover, several studies (e.g., Kuerer et al. [23]) have shown that a minimally invasive biopsy after NAT can be of significant predictive value in these patients, especially in those patients with aggressive tumors and a high probability of pCR after optimal NAT. Therefore, surgery can become less invasive or might be skipped in the future.

This approach might even be used in midterm biopsies for the evaluation of response after NAT to eventually switch to other therapies if there is no response to the initial cycles of therapy. This is a unique opportunity offered only by NACT. An attempt in this direction was done by the GeparTrio study in which patients without an optimal response after initial EC (epirubicin cyclophosphamide) chemotherapy induction who were switched to an alternative regimen had a significant benefit from this therapy [30].

The newest generation of neoadjuvant studies is also including windows of treatment like the GeparNuevo study, proving that the addition of a T-cell checkpoint inhibitor even as a window before NACT might significantly improve pCR and also outcome [31].

The lessons which we have learned in more than 20 years from prospective randomized trials using NACT are summarized in the following:

- Patient selection should be based on tumor biology.

- Imaging of the breast and lymph nodes before and during NACT is mandatory. 
- Optimal systemic therapy should include at least $6 \mathrm{cy}$ cles of anthracyclines with taxanes and a minimum duration of 18 weeks of therapy.

- NACT with anti-HER2 treatment in patients with HER2-overexpressing tumors is mandatory.

- Careful planning of surgery in a multidisciplinary team has to be performed together with specialists in imaging, breast surgeons, pathologists, and radiotherapists.

- This includes tumor clipping before NACT and sentinel node biopsy after NACT.

- The use of standardized pathology protocols for response evaluation in the breast and lymph nodes is crucial.

The critical article by Dediu and Zielinski should not be a reason to do less NAT but encourage us to treat our patients according to the experience and standards we have achieved in the last quarter of the century and to develop even more modern neoadjuvant and postneoadjuvant protocols which will tailor breast cancer treatments even better.

\section{Conclusion}

NAT is the first choice for patients with locally advanced breast cancer. In patients who have a good clinical and imaging response after NACT. Surgery within the new borders of the tumor is safe. Less radical surgery of the breast and axillary lymph nodes is possible, but surgery is still mandatory. In patients with operable breast cancer, NACT is the first choice if tumor biology is aggressive (HER2-neu overexpression, TNBC, luminal B cancer). The prediction of outcome by pCR is possible, especially in patients with HER2-positive or triple-negative tumors. Non-pCR patients with residual disease in the breast and/or in the ipsilateral lymph nodes should be treated with capecitabine if the tumor is HER2 negative (especially TNBC) or with T-DM1 if the tumor is HER2positive. Further studies should address the question of escalation of medical treatment (CDK $4 / 6$ inhibitors in patients with endocrine-responsive tumors, PARP inhibitors in patients with BRCA mutations), or de-escalation of surgery, locoregional radiotherapy, and medical treatment in patients with $\mathrm{pCR}$.

\section{References}

1 Fisher B, Brown A, Mamounas E, Wieand S, Robidoux A, Margolese RG, et al. Effect of preoperative chemotherapy on local-regional disease in women with operable breast cancer: findings from National Surgical Adjuvant Breast and Bowel Project B-18. J Clin Oncol. 1997 Jul;15(7):2483-93.

2 Untch M, Fasching PA, Konecny GE, Hasmüller S, Lebeau A, Kreienberg R, et al. Pathologic complete response after neoadjuvant chemotherapy plus trastuzumab predicts favorable survival in human epidermal growth factor receptor 2-overexpressing breast cancer: results from the TECHNO trial of the AGO and GBG study groups. J Clin Oncol. 2011 Sep;29(25):3351-7.

3 Gianni L, Eiermann W, Semiglazov V, Lluch A, Tjulandin S, Zambetti M, et al. Neoadjuvant and adjuvant trastuzumab in patients with HER2-positive locally advanced breast cancer (NOAH): follow-up of a randomised controlled superiority trial with a parallel HER2-negative cohort. Lancet Oncol. 2014 May;15(6):640-7.

4 Cortazar P, Zhang L, Untch M, Mehta K, Costantino JP, Wolmark N, et al. Pathological complete response and long-term clinical benefit in breast cancer: the CTNeoBC pooled analysis. Lancet. 2014 Jul;384(9938):164-72.

5 Curigliano G, Burstein HJ, P Winer E, Gnant M, Dubsky P, Loibl S, et al.; St. Gallen International Expert Consensus on the Primary Therapy of Early Breast Cancer 2017. Deescalating and escalating treatments for earlystage breast cancer: the St. Gallen International Expert Consensus Conference on the Primary Therapy of Early Breast Cancer 2017. Ann Oncol. 2017 Aug;28(8):1700-12.
6 Untch M, Jackisch C, Schneeweiss A, Conrad B, Aktas B, Denkert C, et al; German Breast Group (GBG); Arbeitsgemeinschaft Gynäkologische Onkologie-Breast (AGO-B) Investigators. Nab-paclitaxel versus solvent-based paclitaxel in neoadjuvant chemotherapy for early breast cancer (GeparSepto-GBG 69): a randomized, phase 3 trial. Lancet Oncol. 2016;17(3):345-56.

7 Schneeweiss A, Jackisch C, Schmatloch S, Aktas B, Denkert C, Schem C, et al. Survival analysis of the prospectively randomized phase III GeparSepto trial comparing neoadjuvant chemotherapy with weekly nab-paclitaxel with solvent-based paclitaxel followed by anthracycline/cyclophosphamide for patients with early breast cancer - GBG69 [abstract GS305]. San Antonio Breast Cancer Symposium 2017.

8 Loibl S, Volz C, Mau C, Blohmer JU, Costa $\mathrm{SD}$, Eidtmann $\mathrm{H}$, et al. Response and prognosis after neoadjuvant chemotherapy in 1,051 patients with infiltrating lobular breast carcinoma. Breast Cancer Res Treat. 2014 Feb; 144(1):153-62.

9 von Minckwitz G, Schmitt WD, Loibl S, Müller BM, Blohmer JU, Sinn BV, et al. Ki67 measured after neoadjuvant chemotherapy for primary breast cancer. Clin Cancer Res. 2013 Aug;19(16):4521-31.

10 Masuda N, Lee SJ, Ohtani S, Im YH, Lee ES, Yokota I, et al. Adjuvant Capecitabine for Breast Cancer after Preoperative Chemotherapy. N Engl J Med. 2017 Jun;376(22):214759.
11 von Minckwitz G, Huang CS, Mano MS, Loibl S, Mamounas EP, Untch M, et al.; KATHERINE Investigators. Trastuzumab emtansine for residual invasive HER2-positive breast cancer. N Engl J Med. 2019 Feb 14; 380(7):617-28.

12 von Minckwitz G, Schneeweiss A, Loibl S, Salat C, Denkert C, Rezai M, et al. Neoadjuvant carboplatin in patients with triple-negative and HER2-positive early breast cancer (GeparSixto; GBG 66): a randomised phase 2 trial. Lancet Oncol. 2014 Jun;15(7):747-56.

13 Loibl S, Untch M, Burchardi N, Huober JB, Blohmer JU, Grischke E-M, et al. A randomized phase II neoadjuvant study (GeparNuevo) to investigate the addition of durvalumab, a PD$\mathrm{L} 1$, to a taxane-anthracycline containing chemotherapy in triple negative breast cancer (TNBC) [abstract]. J Clin Oncol. 2018;Suppl; 104.

14 von Minckwitz G, Kaufmann M, Kümmel S, Eiermann W, Blohmer JU, Costa SD, et al.; GBG and AGO-B study groups. Local recurrence risk after neoadjuvant chemotherapy. Pooled analysis on 5477 breast cancer patients. Cancer Res. 2011;71(24 Suppl):142s.

15 Mamounas EP, Cortazar P,Zhang L, Locoregional recurrence (LRR) after neoadjuvant chemotherapy (NAC): Pooled-analysis results from the collaborative trials in neoadjuvant breast cancer (CTNeoBC) [abstract 61]. ASCO 2014.

16 Swicher SK, Vila J, Tucker SL, et al. Locoregional control according to breast cancer subtype and response to neoadjuvant chemotherapy in breast cancer patients undergoing breast-conserving surgery. Ann Surg Oncol. 2016 Mar;23(3):749-56. 
17 Guidelines of the AGO Breast Committee. https://www.ago-online.de/en/guidelinesmamma/march-2018/.

18 Bossuyt V, Provenzano E, Symmans WF, Boughey JC, Coles C, Curigliano G, et al.; Breast International Group-North American Breast Cancer Group (BIG-NABCG) collaboration. Recommendations for standardized pathological characterization of residual disease for neoadjuvant clinical trials of breast cancer by the BIG-NABCG collaboration. Ann Oncol. 2015 Jul;26(7):1280-91.

19 Kuehn T, Bauerfeind I, Fehm T, Fleige B, Hausschild M, Helms G, et al. Sentinellymph-node biopsy in patients with breast cancer before and after neoadjuvant chemotherapy (SENTINA): a prospective, multicentre cohort study. Lancet Oncol. 2013 Jun 14(7):609-18

20 Boughey JC, Suman VJ, Mittendorf EA, Ahrendt GM, Wilke LG, Taback B, et al.; Alliance for Clinical Trials in Oncology. Sentinel lymph node surgery after neoadjuvant chemotherapy in patients with node-positive breast cancer: the ACOSOG Z1071 (Alliance) clinical trial. JAMA. 2013 Oct;310(14):145561.

21 Caudle AS, Yang WT, Krishnamurthy S, Mittendorf EA, Black DM, Gilcrease MZ, et al Improved Axillary Evaluation Following Neoadjuvant Therapy for Patients With Node-Positive Breast Cancer Using Selective Evaluation of Clipped Nodes: Implementation of Targeted Axillary Dissection. J Clin Oncol. 2016 Apr;34(10):1072-8.
22 Donker M, Straver ME, Wesseling J, Loo CE, Schot M, Drukker CA, et al. Marking axillary lymph nodes with radioactive iodine seeds for axillary staging after neoadjuvant systemic treatment in breast cancer patients: the MARI procedure. Ann Surg. 2015 Feb;261(2):37882.

23 Kuerer HM, Rauch GM, Krishnamurthy S, Adrada BE, Caudle AS, DeSnyder SM, et al. A Clinical Feasibility Trial for Identification of Exceptional Responders in Whom Breast Cancer Surgery Can Be Eliminated Following Neoadjuvant Systemic Therapy. Ann Surg. 2018 May;267(5):946-51.

24 Mamounas EP, Anderson SJ, Dignam JJ, Bear HD, Julian TB, Geyer CE Jr, et al. Predictors of locoregional recurrence after neoadjuvant chemotherapy: results from combined analysis of National Surgical Adjuvant Breast and Bowel Project B-18 and B-27. J Clin Oncol. 2012 Nov;30(32):3960-6.

25 Valachis A, Mamounas EP, Mittendorf EA, Hayashi N, Ishitobi M, Natoli C, et al. Risk factors for locoregional disease recurrence after breast-conserving therapy in patients with breast cancer treated with neoadjuvant chemotherapy: an international collaboration and individual patient meta-analysis. Cancer. 2018 Jul;124(14):2923-30.

26 Mark KS, Harris JR. Radiotherapy issues after neoadjuvant chemotherapy. I Natl Cancer Inst Monogr. 2015 May;2015(51):87-9.
27 Untch M, von Minckwitz G, Gerber B, Schem C, Rezai M, Fasching PA, et al.; GBG and the AGO-B Study Group. Survival Analysis After Neoadjuvant Chemotherapy With Trastuzumab or Lapatinib in Patients With Human Epidermal Growth Factor Receptor 2-Positive Breast Cancer in the GeparQuinto (G5) Study (GBG 44). J Clin Oncol. 2018 May; 36(13):1308-16.

28 Martin M, Holmes FA, Ejlertsen B, Delaloge S, Moy B, Iwata H, et al.; ExteNET Study Group. Neratinib after trastuzumab-based adjuvant therapy in HER2-positive breast cancer (ExteNET): 5-year analysis of a randomised, double-blind, placebo-controlled, phase 3 trial. Lancet Oncol. 2017 Dec;18(12):1688-700.

29 Geyer CE, Loibl S, Mamounas EP, et al: A phase 3, randomized, open-label trial comparing trastuzumab emtansine and trastuzumab as adjuvant therapy for HER2-positive primary breast cancer with residual invasive tumor in the breast or axillary lymph nodes following preoperative therapy (KATHERINE). Cancer Res. 2013;73:(24 Suppl):Abstract nr OT1-1-06.

30 Geyer CE Jr, Huang C-S, Mano MS, Loibl S, Mamounas EP, Untch M, et al. Phase III study of trastuzumab emtansine (T-DM1) vs trastuzumab as adjuvant therapy in patients with HER2-positive early breast cancer with residual invasive disease after neoadjuvant chemotherapy and HER2-targeted therapy including trastuzumab: primary results from KATHERINE [abstract GS1-10]. San Antonio Breast Cancer Symposium (SABCS) 2018.

31 Loibl S, Denkert C. How much information do we really need after neoadjuvant therapy for breast cancer? J Clin Oncol. 2017 Apr; 35(10):1029-30. 